\title{
Perspectivas profissionais da Medicina do Esporte
}

\author{
Arnaldo José Hernandez
}

\section{INTRODUÇÃO}

$A^{\prime}$ Medicina do Esporte é uma especialidade médica globalmente reconhecida e no Brasil tem demonstrado uma presença crescente tanto no aspecto científico quanto no institucional, além de ser um campo profissional estabelecido. Os aspectos médicos da atividade física, estudados pela Medicina do Exercício e do Esporte, ou Medicina Esportiva (ME), e suas áreas complementares, estão cada vez mais presentes na sociedade moderna.

Para citar um exemplo relativamente recente - sedentarismo e a dieta inadequada foram considerados, pela Associação Médica Americana, como uma provável causa de morte mais frequente do que o tabagismo (USA Today, 10 de março de 2004). No ano 2000, a obesidade, que apresenta frequência em ascensão, foi a responsável por cerca de $16,6 \%$ das mortes (400.000 óbitos) nos Estados Unidos da América enquanto $18,1 \%$ das mortes está relacionadas com o tabagismo $(435.000$ óbitos), que mostra taxas decrescentes. Essas causas de óbito são passíveis de prevenção e a $\mathrm{ME}$, entre outras especialidades médicas, age sobre ambas, direta ou indiretamente. A atividade física deve ser vista, antes de tudo, como uma ferramenta das mais importantes em prol da verdadeira medicina preventiva aplicada1,2,3 e a ME como a especialidade responsável por sua supervisão na área médica. A sociedade moderna apresenta novas necessidades: nas áreas da prevenção, da terapêutica, da promoção da saúde, e também na do alto desempenho esportivo. Acompanhando essa tendência e a efetiva expansão desse mercado profissional faz-se necessária a formação de médicos especializados.

Além da atividade física para a população em geral, competições poliesportivas, como Jogos Olímpicos, Pan-Americanos, Nacionais, Regionais e provas de maratona, de triatlo e de aventura, entre outras, é indispensável uma estrutura específica de atendimento, de apoio e de retaguarda aos participantes e às delegações.

Vários países possuem programas de formação na especialidade. Alguns possuem diferentes programas de mestrado e de doutorado, que complementam a formação em distintas especialidades da medicina e das ciências aplicadas ao esporte. Organizações não governamentais, como a Federação Internacional de Medicina Desportiva (FIMS), a Comissão Médica do Comitê Olímpico Internacional (COI), o Colégio Americano de Medicina Esportiva (ACSM), a Sociedade Americana de Ortopedia para Medicina Esportiva (AOSSM) e a Confederação Pan-Americana de Medicina Desportiva (COPAMEDE), cientes da relevância da $M E$, realizam ações para o desenvolvimento da especialidade a nível internacional. A FIMS e a Organização Mundial da Saúde (OMS) colaboram em programas conjuntos para enfrentar as enfermidades crônicas não transmissíveis, nos campos da educação, da promoção, da prevenção e da reabilitação. A Sociedade Brasileira de Medicina do Exercício e do Esporte (SBMEE), criada em 1962 e filiada à Associação Médica Brasileira (AMB), tem mais de 700 especialistas e vem atuando sistematicamente no fortalecimento da especialidade e seu campo de atuação. Como prova disso a criação da Residência Médica em Medicina do Esporte, em 2005. Ações e documentos organizados pela SBMEE, tais como suas várias diretrizes em Medicina Esportiva, contribuem para a compreensão da relevância dessa especialidade médica que envolve profissionais e pesquisadores de diferentes especialidades médicas (medicina do esporte, pneumologia, cardiologia, ortopedia e traumatologia, endocrinologia, pediatria, geriatria, reumatologia, fisiatria, entre outras) e de outras áreas da saúde tais como: fisioterapia, educação física, enfermagem, nutrição, psicologia, para mencionar algumas. 
A clara multidisciplinaridade da Medicina Esportiva torna obrigatória uma visão abrangente dessa área e é natural que cada uma das áreas com interface na especialidade desenvolva atividades em pontos específicos como na Ortopedia e Traumatologia. Por outro lado, é necessário que fique claro o entendimento da ME como uma especialidade médica independente e com seu corpo de conhecimento próprio e definido.

\section{Uma breve história da Medicina do Esporte}

Embora para alguns possa parecer que a ME seja uma especialidade dos tempos modernos, desde a antigüidade ela é citada em diferentes momentos da história da medicina. Há quase 5.000 anos atrás os chineses já descreviam uma série de exercícios recomendados para prováveis diabéticos e orientações nutricionais para promoção da saúde. Os antigos livros da Índia, datados de 1.500 a.C., os livros dos Vedas (Atharva-Veda), também mencionavam diferentes exercícios terapêuticos para a promoção da saúde, conforme descrito no Papírus Ebers. Durante o Período Greco-Romano Heródicus, que é considerado o primeiro médico do esporte, acompanhava os então competidores dos jogos olímpicos da antiguidade. Hipócrates também ressaltava a relevância da atividade física para a saúde. Galeno, em 150 a.C., é tido como o primeiro medico de equipe por cuidar dos gladiadores romanos.

$\mathrm{Na}$ era cristã Hackin Avicenna e Alphonsi Borelii, que escreveu o primeiro texto de fisiologia do aparelho locomotor deram suas importantes contribuições a essa área da medicina. Em 1743, na França, Nicholas Andry que é considerado o pai da ortopedia, ressalta a importância dos exercícios físicos para as crianças e após quase um século o americano Edward Hitchicock cria a primeira escola de Educação Física. A partir do século XX surgem várias organizações que se voltam a essa especialidade médica especialmente após os Jogos Olímpicos de Inverno de Montreaux, na Suíça, quando é fundada a Federação Internacional de Medicina do Esporte (FIMS). Em 1942 é criada a Sociedade Paulista de Medicina Desportiva (SPAMDE) e em 1962 a Federação Brasileira de Medicina do Esporte, o qual passou a ser a Sociedade Brasileira de Medicina do Exercício e do Esporte (SMBEE) em 1998.

Organograma da unidade de medicina esportiva

Embora possamos organizar um Setor de Medicina do Esporte de diferentes formas sugerimos um organograma (Figura 1) que busca contemplar tanto a individualidade da ME, como Especialidade Médica independente, como o reconhecimento das intersecções das diversas especialidades médicas e áreas da saúde que com ela se relacionam. É necessário que se diga que a atenção nessa especialidade também envolve 0 atendimento em todas as faixas etárias, da criança ao idoso, tanto da população geral quanto de atletas de alto desempenho.

Para fins de organização de uma Unidade de ME consideramos que sejam cinco os setores que devem ser estruturados: o de Clínica do Exercício e do Esporte, o de Ortopedia e Traumatologia do Exercício e do Esporte, o de Avaliação Funcional do Exercício e do Esporte, o de Reabilitação no Exercício e no Esporte e o das Áreas de Suporte ao Exercício e ao Esporte.

\section{Setores de atuação na Medicina do Esporte}

\section{Clínica Médica do Exercício e do Esporte}

Tanto o indivíduo da população em geral que busca, de forma espontânea ou por encaminhamento, orientação para atividade física quanto o esportista de alto desempenho devem receber, à princípio, a atenção de médicos especialistas para esse fim (médico do esporte), e com suporte de retaguarda nas diferentes especialidades médicas para situações eventuais de maior complexidade. Esse consiste no ambulatório geral de ME. Os problemas de atenção primária e secundária são abordados por esse especialista que, além da avaliação clínica solicita os exames complementares e as avaliações funcionais que julgar necessárias para a situação ${ }^{4,5}$. Após essas avaliações cabe a esse setor realizar o seguimento desses indivíduos ao longo de seus treinamentos. Mesmo quando esses profissionais são também especialistas em outras áreas, a eles cabe a função de realizar esse tipo de atendimento quando imbuídos dessa função.

Além desse primeiro contato, cabe a esse médico a vivência do dia-a-dia do esporte, junto às equipes e aos atletas, supervisionando seu treinamento, realizando prevenção de problemas clínicos e ortopédicos, oferecendo suporte nutricional e psicológico em situações de atendimento inicial, em conjunto com as áreas afins, e atendendo intercorrências médicas como o caso extremo de morte súbita e sua prevenção6,7 durante eventos esportivos e viagens de delegações. Nessas atividades o especialista também participa do apoio ao treinamento físico e técnico das diferentes modalidades esportivas. 


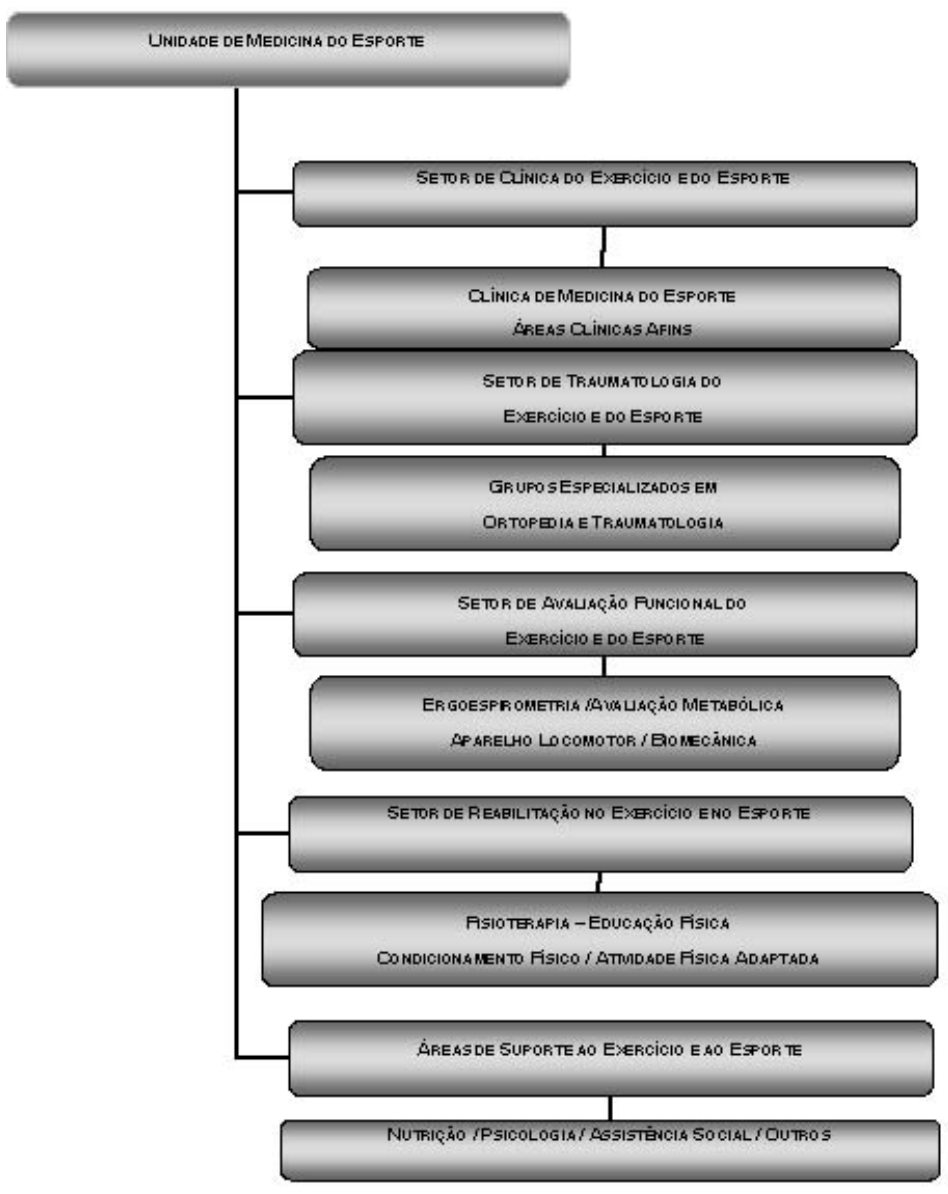

Figura 1. Organograma de um Setor de Medicina do Esporte

\section{Setor de Ortopedia e Traumatologia do Exercício e do Esporte}

As lesões esportivas do aparelho locomotor, típicas ou eventuais, acontecem com grande freqüência, tanto na prática da atividade física geral quanto no esporte de alto rendimento ${ }^{8-13}$. Esse setor é responsável pela atenção clínica e cirúrgica, nessa especialidade, dos pacientes em tais condições. Esse atendimento deve, preferencialmente, ser realizado por médicos com a dupla formação - em medicina do esporte e em ortopedia e traumatologia. Isso permite a assistência global ao cliente e a compreensão de suas necessidades como praticante da atividade física. Ainda, no caso do alto desempenho e durante o tratamento da lesão esportiva, a reorientação da atividade física, como o repouso ativo, e a manutenção do condicionamento físico exigem um atendimento integrado com as diferentes áreas afins. Os programas de prevenção de lesões são fundamentais nessa área ${ }^{14,15,16}$.

Nesse ponto é necessário que se considere a situação de uma nova área dentro de uma especialidade já com tradição no mercado profissional como, por exemplo, a Ortopedia e Traumatologia. Em nosso meio, histórica e culturalmente, essa especialidade tem suas subespecialidades definidas por regiões anatômicas. Nos Estados Unidos da América essa subdivisão é definida pelo tipo de procedimento a ser realizado. $\mathrm{Na} \mathrm{ME}$, os chamados procedimentos de partes moles e cirurgias artroscópicas são geralmente realizados por ortopedistas que atuam em mais de uma articulação como ombro e joelho, os quais são também considerados os traumatologistas do esporte.

\section{Setor de Avaliação Funcional do Exercício e do Esporte}

As atividades desse setor abrangem a avaliação e o acompanhamento de indivíduos envolvidos com a atividade física, em seus diferentes níveis, por meio de testes de função das variáveis e características fisiológicas durante o esforço físico, tais como: testes ergoespirométricos, curvas de lactato, testes neurofuncionais e flexibilidade, entre outros. Outras formas de avaliação de parâmetros relevantes para 
a prescrição e o acompanhamento de atividade física e treinamento, como a avaliação da composição corporal, a avaliação da força muscular, a avaliação da postura, a biomecânica dos gestos esportivos e a toxicologia também são de sua responsabilidade ${ }^{17}$. São ainda atividades desse setor as avaliações de campo, que correspondem basicamente às avaliações já mencionadas no item anterior, obtidas, porém, durante a prática das atividades físicas específicas, tais como: corrida de velocidade ou de longa duração, saltos, natação e esportes de campo e quadra, entre outras modalidades esportivas.

\section{Setor de Reabilitação no Exercício e no Esporte}

A reabilitação é uma das grandes áreas da ME. Tanto as afecções clínicas como as cirúrgicas dependem diretamente dessa área para o sucesso da recuperação funcional dos pacientes. A manutenção do condicionamento físico é fundamental no período de reabilitação e permite que seja realizada a ponte entre a terapêutica implementada e o retorno mais seguro à atividade física. Na reabilitação, os programas de reabilitação cardíaca e pulmonar são essenciais para as ações de prevenção secundária.

Outra área relacionada a reabilitação relacionada é a da atividade física adaptada, que permite a portadores de deficiência física se beneficiarem com a atividade física, além de dar apoio ao esporte competitivo para deficientes, 0 esporte paraolímpico ${ }^{18}$.

\section{Áreas de Suporte ao Exercício e ao Esporte}

No contexto multidisciplinar, A nutrição voltada à saúde ou para a melhora do desempenho faz parte da atenção global da população em geral e de atletas. Está claro que a orientação nutricional é inerente a qualquer atenção clínica, mas, quando vinculada à promoção da atividade física, é mais eficiente e promove maior adesão ao controle nutricional. No esporte de alto desempenho a suplementação nutricional é desejável e a utilização de recursos ergogênicos lícitos e seguros, uma necessidade ${ }^{19,20}$. Da mesma forma os efeitos psicológicos da prática da atividade física na infância, na vida adulta e na terceira idade, e a sua atenção, não são desprezíveis antes, durante e depois do período competitivo. Outro exemplo de área afim é a assistência social, que cumpre papel relevante, particularmente nas classes sociais menos favorecidas, onde a atividade física e o esporte podem ser a diferença entre a criminalidade e a inclusão social. A enfermagem também tem papel relevante em todos os setores mencionados e a integração entre todos esses profissionais é de grande relevância para a atenção global à comunidade, e aos atletas de per si.

\section{Inserção profissional}

A clara multidisciplinaridade da Medicina do Exercício e do Esporte deixa patente que, nessa área, o trabalho integrado de diferentes profissionais é obrigatório e as boas relações éticas devem reger essas ações. Naturalmente a especialidade em Medicina do Esporte é uma prerrogativa da área médica, enquanto especialidade médica assim definida. Em todos os grandes eventos esportivos no mundo, as Clínicas do Esporte são coordenadas por médicos do esporte ${ }^{21} \mathrm{e}$ todas as áreas trabalham de forma integrada uma vez que existem algumas situações onde a formação médica não nos permite atuar mas, sem dúvida, a formação do médico é a mais abrangente nesse sentido.

O mercado de trabalho para o médico, nessa especialidade, é evidente havendo grande carência desses profissionais para exercer suas funções em clubes, equipes, agremiações esportivas e mesmo em escolas e no Programa de Saúde da Família, onde o estímulo e orientação adequada de atividade física deve ser parte obrigatória dos programas de promoção da saúde. O envolvimento desse especialista em todas essas ações mais do que recomendável deve ser mandatório por razões técnicas, profissionais e, sobretudo, éticas. No Brasil, a perspectiva de realização de uma Copa do Mundo e dos Jogos Olímpicos cria uma situação favorável à visibilidade dessa especialidade, e seu estabelecimento definitivo como uma grande área para a atuação profissional dos médicos com essa formação.

\section{REFERÊNCIAS}

1. Morris JN, Heady JA, Raffle PA, Roberts CG, Parks JW. Coronary heart-disease and physical activity of work. Lancet. 1953;265:1053-7.

2. Paffenbarger RS, Jr., Hyde RT, Wing AL, Hsieh CC. Physical activity, all-cause mortality, and longevity of college alumni. N Engl J Med. 1986;314(10):605-13.

3. Chodzko-Zajko WJ, Proctor DN, Fiatarone Singh MA, Minson CT, Nigg CR, Salem GJ, et al. American College of Sports Medicine position stand. Exercise and physical activity for older adults. Med Sci Sports Exerc. 2009;41(7):1510-30.

4. Maron BJ, Thompson PD, Puffer JC, McGrew CA, Strong WB, Douglas PS, et al. Cardiovascular preparticipation screening of competitive athletes. A statement for health professionals from the Sudden Death Committee (clinical cardiology) and Congenital 
Cardiac Defects Committee (cardiovascular disease in the young). American Heart Association. Circulation. 1996;94(4):850-6.

5. Pelliccia A, Culasso F, Di Paolo FM, Accettura D, Cantore R, Castagna W, et al. Prevalence of abnormal electrocardiograms in a large, unselected population undergoing pre-participation cardiovascular screening. Eur Heart J. 2007;28(16):2006-10.

6. Maron BJ, Gohman TE, Aeppli D. Prevalence of sudden cardiac death during competitive sports activities in Minnesota high school athletes. J Am Coll Cardiol. 1998;32(7):1881-4.

7. Corrado D, Basso C, Rizzoli G, Schiavon M, Thiene G. Does sports activity enhance the risk of sudden death in adolescents and young adults? J Am Coll Cardiol. 2003;42(11):1959-63.

8. Beutler Al, Marshall SW. Epidemiology and prevention of sports injuries". In: Garrick JG. OKU, Sports Medicine, 2004.

9. Foster, C, Porcari, JP, Battista, RA, Udermann, B, Wright, G, Lucia, A. The Risk in Exercise Training. Am J Lifestyle Med. 2008;2(4):279-84.

10. Sorensen L, Larsen SE, Rock ND. The epidemiology of sports injuries in school-aged children. Scand J Med Sci Sports. 1996;6(5):281-6.

11. Kokron AEV, Hernandez AJ, Costa AJF, Bucalem G, Longo $\mathrm{CH}$, Rosemberg LA, et al. Importância da ressonância magnética na avaliação das lesões traumáticas da placa de crescimento epifisária. Rev Bras Ortop. 1996;31(5):435-40.

12. Schneider S, Seither B, Tonges S, Schmitt H. Sports injuries: population based representative data on incidence, diagnosis, sequelae, and high risk groups. $\mathrm{Br}$ J Sports Med. 2006;40(4):334-9.

13. Darrow CJ, Collins CL, Yard EE, Comstock RD. Epidemiology of severe injuries among United States high school athletes: 2005-2007. Am J Sports Med.
2009;37(9):1798-805.

14. Van Tiggelen D, Wickes S, Stevens V, Roosen $P$, Witvrouw E. Effective prevention of sports injuries: a model integrating efficacy, efficiency, compliance and risk-taking behaviour. Br J Sports Med. 2008;42(8):648-52.

15. Griffin LY, Agel J, Albohm MJ, Arendt EA, Dick RW, Garrett WE, et al. Noncontact anterior cruciate ligament injuries: risk factors and prevention strategies. J Am Acad Orthop Surg. 2000;8(3):141-50.

16. Hernandez AJ, Amatuzzi MM, Albuquerque RFM, Laraya MH, Rubin MLL, Martinelli M Filho. Estudo prospectivo controlado do tratamento das lesões isoladas do ligamento cruzado anterior com reconstrução e osteomias concomitantes. Rev Bras Ortop. 2004;39(7):371-81.

17. Santos Silva PR, Greve JM, Hernandez AJ. Effect of interval time at ventilatory sampling in variability of maximum oxygen uptake in Professional soccer player. J Sci Sports Med.

18. Hernandez AJ, Rezende MU. Atividade física e condições ortopédicas limitantes. In: Gorgatti MG, Costa RF. Atividade física adaptada. São Paulo: Manole; 2005. p.248-85.

19. Favano A, Santos-Silva PR, Nakano EY, Pedrinelli A, Hernandez AJ, Greve JM. Peptide glutamine supplementation for tolerance of intermittent exercise in soccer players. Clinics (São Paulo). 2006;63(1):27-32.

20. Hernandez AJ, Nahas RM, Rodrigues TR, Meyer F, Zogaib P, Lazzoli JK, et al. Modificações dietéticas, reposição hídrica suplementos alimentares e drogas: comprovação de ação ergogênica e potenciais riscos para a saúde. Rev Bras Med Esporte. 2009;15(Suppl.3):3-11.

21. Brukner P, Khan K. Sports medicine: the team approach. In: Brukner $\mathrm{P}$, editor. Clinical sports medicine. Australia: McGraw-Hill; 2005. 